\title{
Stage IIIB Cervical Cancer AJCC v8
}

National Cancer Institute

\section{Source}

National Cancer Institute. Stage IIIB Cervical Cancer A/CC v8. NCI Thesaurus. Code C139752.

Stage IIIB includes: T3b, Any N, M0. T3b: extending to pelvic wall and/or causing hydronephrosis or nonfunctioning kidney. M0: No distant metastasis. (AJCC 8th Ed.) 